\title{
Online-Gym: multiuser virtual gymnasium using RINIONS and multiple Kinect devices
}

\author{
Fernando Cassola \\ INESC TEC \\ Porto, Portugal \\ fernandocassola@gmail.com
}

Sílvia Ala

UTAD, Universidade de Trás-os-Montes e Alto Douro
Vila Real, Portugal
silviaala@sapo.pt

Fausto de Carvalho

Portugal Telecom Inovação e Sistemas

Aveiro, Portugal

cfausto@telecom.pt

\author{
Hugo Paredes, Benjamim Fonseca, Paulo Martins \\ INESC TEC \\ UTAD, Universidade de Trás-os-Montes e Alto Douro \\ Vila Real, Portugal \\ hparedes@utad.pt,benjaf@utad.pt,pmartins@utad.pt
}

\author{
Francisco Cardoso \\ LabPEC - Laboratório de Psicologia Experimental Clínica \\ UTAD, Universidade de Trás-os-Montes e Alto Douro \\ line 3-City, Country \\ fcardoso@utad.pt \\ Leonel Morgado \\ INESC TEC \\ Universidade Aberta \\ Lisbon, Portugal \\ leonel.morgado@uab.pt
}

\begin{abstract}
To enhance older citizen's practice of physical exercise, we present the architecture, development, and pilot testing of a multiuser online gymnasium based on Kinect motion capture and OpenSimulator, which aims to enable socialization and supervision of exercise practice without travel requirements. The prototype was tested simultaneously with 4 elders at different locations, providing data on the feasibility of the approach and informing subsequent development and research.
\end{abstract}

Keywords-elders; exergames; OpenSimulator; Kinect

\section{INTRODUCTION}

Computational platforms for physical activity can promote the physical well-being and mental health of older people [1]. From this concept, we idealized, developed, and tested a prototype three-dimensional multiuser online gymnasium environment. Physical exercise can thus be made at home enjoying the socialization aspects of team gymnastics, unlike the lonely experience of exercise videos. It has been known that this can be a key factor to engage populations with these professionals [2]. It also enables the possibility of remote support by trainers, teachers, or therapists, who become able to visualize the ongoing exercises from various perspectives. Our approach is targeted at elders, particularly those finding it difficult to exercise regularly in groups, due to living in rural areas, troublesome urban neighbourhoods or personal locomotion issues. Our Online-Gym approach involved the use of multiple Microsoft Kinect devices for simultaneous multiuser interaction in virtual spaces [3]. In practice, different users, physically apart, may attend a gymnastics session over

This work was funded by Portugal Telecom Inovação under the Innovation Plan of the PT Group, and by FEDER funds through the Programa Operacional Fatores de Competitividade - COMPETE and National Funds through FCT - Foundation for Science and Technology under the project «FCOMP - 01-0124-FEDER-022701». the Internet, being represented as avatars animated by their Kinect-captured motions in front of personal computers. In this paper we present the prototype, which we called Online-Gym, its architecture and implementation, and early user testing.

\section{INTERACTIVE TECHNOLOGIES AND PHYSICAL ACTIVITY}

A limitation of traditional approaches to promote physical activity remotely is the generic nature of exercises and the lack of feedback [4]. A new approach which has been gaining traction in this regard is the use of videogames [5]. Particularly, due to various low cost technological devices which enable motion to be captured, so that users can provide their gestures as input to exercise platforms, a development which is embodied in the term "exergames" [6][7]. We are referring to devices such as the Nintendo Wii [8], the Xbox with Kinect [9] and PlayStation Move [10]. Self-motivation for exercising alone is hard [11], and exergames may contribute to increase the level of exercise practice by elders, by combining social interaction with online gymnastics [3][11][12]. But benefits may also arise for gymnastics trainers' education and practice. As Morgado et al. put forward [13], when online multiuser spaces become the location of real (rather than simulated) activities, one can include them in on-the-job training and professional traineeships. That is, students of physical therapy, gymnastics or other similar areas could remotely participate in sessions online with supervisory professionals and gymnastics participants, enlarging the scope of contexts and opportunities for participating in his/her future professional practice. 


\section{ONLINE-GYM PROTOTYPE OVERVIEW}

\section{A. Avatar puppeteering through motion capture}

Several projects and libraries provide motion capture services for Kinect devices. Two low-level libraries provide the basic services and are readily available: Microsoft Kinect SDK [14] and OpenNI [15]. On top of the raw data provided by these libraries, several middleware projects emerged for processing gestures and motion. In a previous work, we compared three of them: RINIONS, FAAST, and NUILIB [16], and selected RINIONS for Online-Gym because it provides the basic services of capturing and relaying animation data already in the form of an avatar skeleton.

\section{B. RINIONS: remote transmission of motion}

RINIONS was originally known as SLKinect2 [17], a software package developed at the Tokyo University of Information Sciences. Its focus is transferring user motion as detected by Kinect into client software ("viewer") of virtual worlds using Second Life or OpenSimulator technology, and relaying them remotely. It is available as open source code [18]. It is based on using two pieces of software alongside viewers and servers of Second Life/OpenSimulator. The first is a client that captures user motion using the Kinect SDK or OpenNI. The other is a server that receives and relays motion data among clients (Fig. 1). The clients inject both locallycaptured motion and remotely-received motion into the local user's Second Life/OpenSimulator viewer, by converting Kinect skeletal data into Second Life/OpenSimulator skeleton positions, and then storing them into a shared memory location. Modified versions of the Second Life/OpenSimulator viewers read skeletal position data for a specific animation file from this shared memory location, thus rendering motion data that is continually being updated by the RINIONS client.

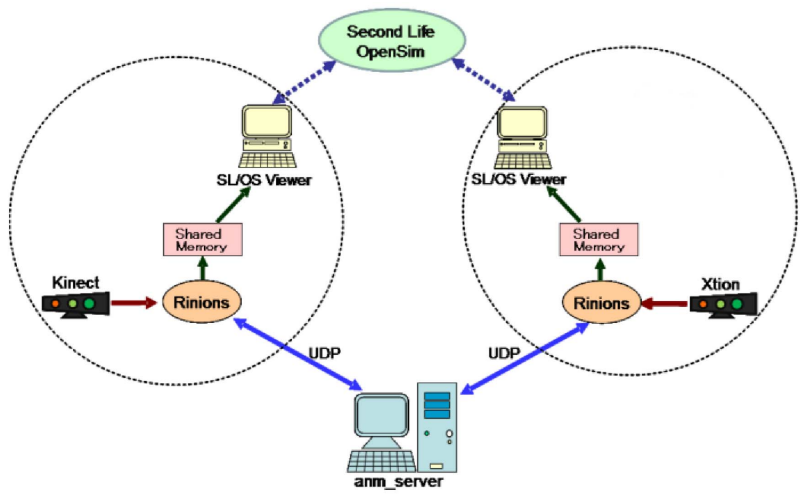

Fig. 1. RINIONS architecture (adapted from [16]).

\section{MINIMIZE LATENCY EFFECTS, CONTRIBUTING TO SYNCHRONIZE PARTICIPANTS' MOTION CAPTURE}

\section{A. Approach summary}

The RINIONS server receives the motion capture frames from the RINIONS clients and relays them in a first-in, firstout basis. Due to the variability in network conditions, this may lead to significant lack of synchrony between users reproducing the same gymnastics activities. In order to tackle this issue, we implemented a prototype version of the server, with the intent of minimizing these issues. At the time of writing, operational tests confirmed its feasibility, but performance tests are still underway.

In our approach, we prioritized the distribution of motion capture frames, dropping some of them when relaying to slower clients. For this purpose, we took advantage of unused bytes in the RINIONS network package format, and used them for tracking quality of service. This implied modifications in both the client and server software of RINIONS, but the protocol packages are still accepted by non-modified RINIONS clients and servers.

\section{B. Modified RINIONS client}

In order to measure the network delay between a RINIONS client and its animation server, our modified RINIONS client takes advantage of available bytes in the RINIONS network package. As shown in Fig. 2, our client includes the local timestamp in each package, using the 3rd and 4th bytes, which were previously identified as "Timer". This data can be used by servers to measure client upload time and its variation. Also, our client in parallel performs ping requests to the RINIONS server, averages their results (in milliseconds) and includes this data in each animation package sent to the server, using two previously unused bytes, which the original protocol had reserved for "other uses". These are labelled as "Ping Average" in Fig. 2. This data can be used by the server to ascertain the animation download time and variation at the client.

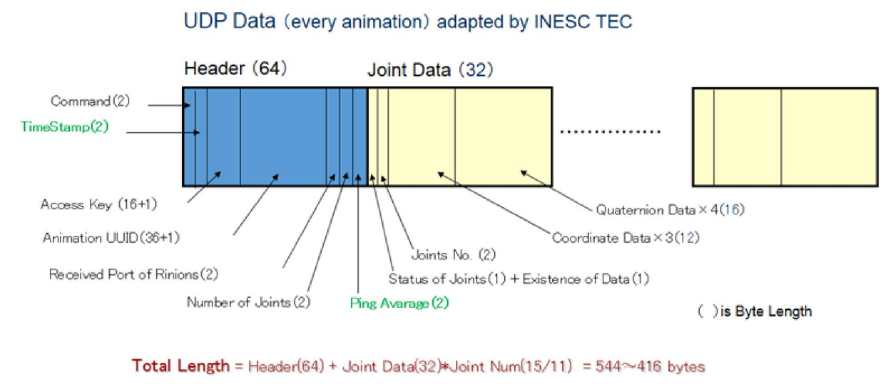

Fig. 2. RINIONS UDP package structure, adapted for Online-Gym.

\section{Modified RINIONS server}

At the server side, our modified RINIONS server processes the new data sent from the modified client. In the current prototype, we are only processing the timestamp data, i.e., reacting to variations in client upload delay. In the future, we intend to evolve the server in order to react also to client download delay.

By looking at the various clients' timestamps, we employed a queuing method to sort the received motion capture frames, before relaying them to the clients. Specifically, upon receiving a RINION client's UDP package containing a motion capture frame, the server places relevant quality of service data in an array (id, IP number, timestamp, and average ping value). That array is then sorted using the timestamp, and the results used to relay only the most recent yet-to-be-relayed motion capture frames. This way, the slower clients always receive only the most recent frames from the faster clients, and this selection of 
constantly updated with each frame uploaded by the clients to the server. The rationale is that since at each machine its locally-captured frames are sent directly by the RINIONS client to the rendering viewer, the minimal delay for other (remote) clients is found in the latest frames sent by those clients.

\section{TESTING WITH END USERS}

\section{A. Test setup}

The prototype was initially submitted to limited in-house operational tests: two computers and two Kinect devices connected to different WiFi networks over the same physical infrastructure. Subsequently, we conducted a limited end-user essay with four elders and a gymnastics teacher, located at four different locations in Portugal, using home DSL connections.

The small number of participants in this essay means that results cannot be generalized to the population in general, but rather seen as early data for qualitative analysis. The observational goals were to a) identify the users' perspectives on the usefulness of such a 3D platform; b) ascertain user satisfaction from participation in the activities; c) gather data on adequacy of image quality, screen size, and sound quality; d) identify hurdles using the Online-Gym platform; e) gather early data on user immersion and presence; f) ascertain whether there was a notion of being in the presence of other users (visible through their avatars); h) ascertain the motivation for further use of the Online-Gym platform; i) gather generic data on deployment and use of the platform.

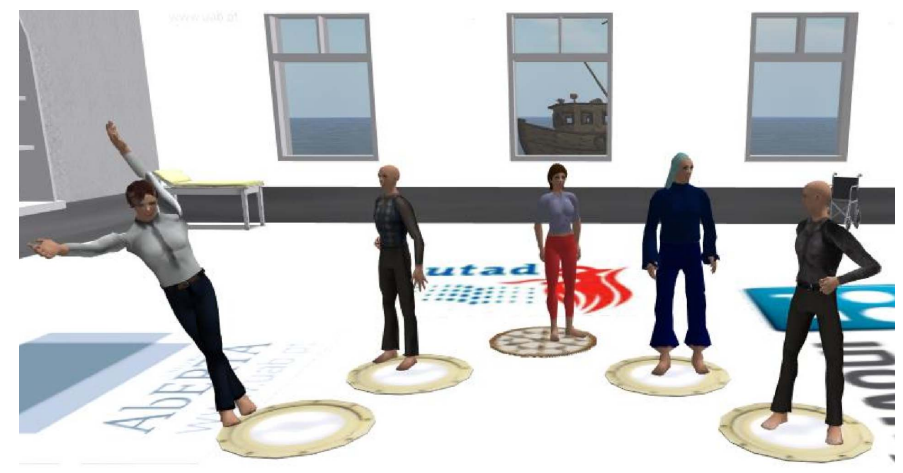

Fig. 3. Online-Gym test session - setup.

At each testing site, a computer was setup with microphone, normal-sized monitor (i.e., 17" to 21"), speakers, a Kinect device, WiFi connection to a domestic Internet router (DSL), RINIONS client software and a RINIONS-modified Second Life/OpenSimulator viewer software. The OpenSimulator server and animation server were running on an Azure cloud.

To perform the test, we initially set up the OpenSimulator viewer by logging into the Online-Gym server with the users' avatars, placing them side-by-side on the virtual gym. Then we established a Skype conference call for voice communication during the session. Finally, we moved the OpenSimulator camera perspective to a frontal position, which enabled participants to see both their own avatar, those of other participants, and that of the gymnastics teacher (Fig. 3).

\section{B. Participants and instruments}

The test participants were invited to participate, and did so willingly. They were located in four towns in Portugal: Bragança (B), Vila Real (V), Aveiro (A), and Ansião (N), road distances varying from $100 \mathrm{~km}$ to $330 \mathrm{~km}$. The participants were: a 67 year-old male; a 73 year-old male; a 65 year-old male; and an 81 year-old female. We employed a questionnaire with items related to the participants' personal characterization, including health condition, attitudes towards exercising and gymnasium use, and prior experience using computers and the Internet. We also included items specifically targeting the Online-Gym essay, using Likert scales and open-ended answers to explore the subjectivity of each participant's viewpoints. Photos and videos were also collected at each site, for further documentation, as well as observation reports.

\section{Results and discussion}

The Online-Gym prototype was operational throughout the user tests, so the collected data refers entirely to the user experience. At the functional level, the observers reported as most pressing issues the following:

- some of the faster user gestures were not properly captured, e.g., opening and closing arms, rotating the torso, repositioning oneself;

- some gestures that were both slow and with low amplitude would often not be captured;

- some gestures are not identified at all, regardless of speed or amplitude, e.g., shoulder pad rotations.

As an observed consequence, sometimes both the gymnastics teacher and/or participants would be unsure of whether a move was being performed by all or not, since some avatars would appear still or jittery in these circumstances. This could be disruptive, with voice calls for confirmation (e.g., "Is everyone ready/OK"?; "Can we start" when the exercise was already ongoing). At least with the older participant, it was also noticeable that there was not awareness from the gymnastics teacher regarding tiredness level, since lack of motion could be interpreted as lack of responsiveness from the system, instead of actual lack of motion from a participant. This holds potential for hazardous consequences, and requires further exploration.

Sound quality was another relevant issue. As mentioned above, we employed a Skype conference call with normal desktop speakers. However, while this might be acceptable in quieter environments, in the course of gymnastics users were some steps back from the computers and speakers. In one case, the location was a seniors' home hall, which albeit empty at the time meant that the sound would not be as audible as desired. Compounded with the participant's age and audition, this required researchers to intervene in the process occasionally to clarify the voice commands and feedback - so further attention also needs to be given to this aspect.

In terms of the actual dynamics of the gymnastics session, a core issue was the occasional difficulty of perceiving details of the intended gesture, motion, or body position. There were two specific issues: sometimes, the size of avatars on the screen was simply too small when seen as a group, and we had to 
reposition the viewer camera so that only two or three avatars (including the teacher and the participant) were visible, for greater size; other times, the exercise required a lateral perspective to be properly understood, and it was necessary to use the traditional computer controls to enact that change in perspective. A complementary aspect of this issue was that sometimes users would move from their position while performing the gymnastics, i.e., advancing or retreating. This impacted their own avatar's position in the virtual world, sometimes ending up in front or behind another user's avatar. These issues impacted the elders' ability to perceive the intended motions immediately, but also the gymnastics teacher's ability to be aware of positioning or motion errors of participants in order to be able to provide adequate feedback.

\section{LESSONS LEARNED AND RECOMMENDATIONS}

Following the tests described above, several issues were only overcome due to the presence of researchers at each site. Specifically, changes of viewer camera perspective need to be automated or at least be streamlined, in order to prevent exercise disruption. Also, avatar positioning is critical in order for a single camera angle to capture at least the participant's avatar and the gymnastics' teacher.

Sound quality in the actual user environment is key for a smooth process, as is the need to accommodate doubts and/or needs for clarify gestures, intent, or physical condition. Harvesting local environment conditions can help, but new training/monitoring exercise procedures may be needed in order to accommodate the context of this new medium.

Screen size is clearly an issue. Even with adequate camera positioning, sometimes avatars would simply be too small to enable a clear identification of gestures. More research is needed on this topic. For instance, to see if a large living room television set would be enough or whether successful interaction would require video projection.

Future studies should take these issues in consideration when exploring alternatives for motion capture, interaction modalities, user interfaces, and technical support needs.

We can draw encouragement from the mostly positive feedback provided by users. The willingness to use this platform again, expressed spontaneously even before questionnaire filling, is an indicator of satisfaction, even in face of the above issues.

\section{ACKNOWLEDGMENTS}

We would like to express our thanks to the cooperation and participation of all end users, plus the local facilitators and the gymnastics teacher.

\section{REFERENCES}

[1] N. L. Atkinson and R. S. Gold, "The promise and challenge of eHealth interventions," American Journal of Health Behavior, vol. 26, pp. 494 503, 2002.

[2] H. Patrick and A. Canevello, "Methodological Overview of A SelfDetermination Theory-Based Computerized Intervention to Promote Leisure-Time Physical Activity," Psychol Sport Exerc, vol. 12, pp. 1319, Jan 2011

[3] F. Cassola, L. Morgado, F. de Carvalho, H. Paredes, B. Fonseca, and P. Martins, "Online-Gym: a 3D virtual gymnasium using Kinect interaction," Procedia Technology, 13, pp. 130-138, 2014.

[4] B. H. Marcus, D. M. Williams, P. M. Dubbert, J. F. Sallis, A. C. King, A. K. Yancey, B. A. Franklin, D. Buchner, S. R. Daniels, and R. P. Claytor, "Physical Activity Intervention Studies: What We Know and What We Need to Know: A Scientific Statement From the American Heart Association Council on Nutrition, Physical Activity, and Metabolism (Subcommittee on Physical Activity); Council on Cardiovascular Disease in the Young; and the Interdisciplinary Working Group on Quality of Care and Outcomes Research," Circulation, vol. 114, pp. 2739-2752, 2006

[5] B. H. Marcus, C. R. Nigg, D. Riebe, and L. H. Forsyth, "Interactive communication strategies: implications for population-based physicalactivity promotion," American Journal of Preventive Medicine, vol. 19, pp. 121-126, 2000.

[6] C. A. O. Vaghetti and S. S. d. C. Botelho, "Virtual learning environments in physical education: a review of the use of Exergames," Ciências \& Cognição 2010, vol. 15, pp. 76-88, 2010.

[7] J. Sinclair, P. Hingston, and M. Masek, "Considerations for the design of exergames," in GRAPHITE '07 - 5th International Conference on Computer Graphics and Interactive Techniques in Australasia and Southeast Asia, Perth, Western Australia, 2007, pp. 289-295.

[8] Nintendo. (30-04-2014). Wii Accessories. Available: http://www.nintendo.co.uk/Wii/Accessories/Accessories-Wii-NintendoUK-626430.html

[9] Microsoft. (30-04-2014). Kinect for Windows. Available: http://www.microsoft.com/en-us/kinectforwindows/

[10] S. C. Entertainment. (30-04-2014). PlayStation Move. Available http://playstationmove.com/

[11] E. Brox, L. F. Luque, G. J. Evertsen, and J. E. G. Hernández, "Exergames for elderly: Social exergames to persuade seniors to increase physical activity," presented at the Pervasive Computing Technologies for Healthcare (PervasiveHealth) 2011, 5th International Conference on, 23-26 May 2011, 2011.

[12] I. Wijnand, N. Henk Herman, K. Yvonne de, and P. Karolien, "Digital game design for elderly users," in Proceedings of the 2007 conference on Future Play \%@978-1-59593-943-2, ed. Toronto, Canada: ACM, 2007, pp. 17-22.

[13] L. Morgado, J. Varajão, D. Coelho, C. Rodrigues, C. Sancin, V. Castello, "The Attributes and Advantages of Virtual Worlds for Real World Training," The Journal of Virtual Worlds and Education, 1 (1) 2010.

[14] Microsoft (30-04-2014). KINECT for Windows | Dev Center. Available: http://www.microsoft.com/en-us/kinectforwindowsdev/start.aspx

[15] OpenNI (30-04-2014). OpenNI. Available: http://github.com/OpenNI/OpenNI

[16] F. Cassola, L. Morgado, H. Paredes, B. Fonseca, P. Martins, and F. de Carvalho, "Online Gym: um ginásio virtual 3D integrando a Kinect análise comparativa de bibliotecas de suporte," in Atas da Videojogos 2013 - Universidade de Coimbra, Portugal, in press.

[17] F. Iseki (30-04-2014), SLKinect2 [Kinect 2 SecondLife Viewer Project]. Available: http://www.nsl.tuis.ac.jp/xoops/modules/xpwiki/?SLKinect2

[18] F. Iseki (30-04-2014), Rinions [RINIONS]. Available: http://www.nsl.tuis.ac.jp/xoops/modules/xpwiki/?Rinions 